\title{
SoFAST: Automated Flare Detection with the PROBA2/SWAP EUV Imager
}

\author{
K. Bonte · D. Berghmans • A. De Groof · K. Steed • \\ S. Poedts
}

Received: 25 May 2012 / Accepted: 10 October 2012

(C) Springer Science+Business Media Dordrecht 2012

\begin{abstract}
The Sun Watcher with Active Pixels and Image Processing (SWAP) EUV imager onboard PROBA2 provides a non-stop stream of coronal extreme-ultraviolet (EUV) images at a cadence of typically 130 seconds. These images show the solar drivers of space-weather, such as flares and erupting filaments. We have developed a software tool that automatically processes the images and localises and identifies flares. On one hand, the output of this software tool is intended as a service to the Space Weather Segment of ESA's Space Situational Awareness (SSA) program. On the other hand, we consider the PROBA2/SWAP images as a model for the data from the Extreme Ultraviolet Imager (EUI) instrument prepared for the future Solar Orbiter mission, where onboard intelligence is required for prioritising data within the challenging telemetry quota. In this article we present the concept of the software, the first statistics on its effectiveness and the online display in real time of its results. Our results indicate that it is not only possible to detect EUV flares automatically in an acquired dataset, but that quantifying a range of EUV dynamics is also possible. The method is based
\end{abstract}

PROBA2 - First Two Years of Solar Observation

Guest Editors: David Berghmans, Anik De Groof, Marie Dominique, and Jean-François Hochedez

K. Bonte $(\bowtie) \cdot$ K. Steed $\cdot$ S. Poedts

Centre for mathematical Plasma-Astrophysics, Department of Mathematics, KU Leuven,

Celestijnenlaan 200B, 3001 Leuven, Belgium

e-mail: katrien.bonte@wis.kuleuven.be

K. Steed

e-mail: kimberley.steed@ wis.kuleuven.be

S. Poedts

e-mail: stefaan.poedts@wis.kuleuven.be

D. Berghmans

Royal Observatory of Belgium, Ringlaan 3, 1180 Brussel, Belgium

e-mail: david.berghmans@oma.be

A. De Groof

European Space Agency, c/o Royal Observatory of Belgium, Ringlaan 3, 1180 Brussel, Belgium

e-mail: anik.degroof@esa.int 
on thresholding of macropixelled image sequences. The robustness and simplicity of the algorithm is a clear advantage for future onboard use.

Keywords Sun: observations - Sun: flares - Sun: instrumentation and data management . Sun: EUV · Sun: corona $\cdot$ Techniques: image processing $\cdot$ PROBA2 $\cdot$ SWAP

\section{Introduction}

Solar flares are among the most violent energy releases in the heliosphere. They can release enormous amounts of free magnetic energy as radiation spread over the entire electromagnetic spectrum. Flares are frequently associated with filament eruptions and coronal mass ejections (CMEs), while large flares are often the source of solar energetic particles (Benz, 2008). Flares are therefore at the origin of the most harmful space-weather effects in the space environment of the Earth and are thus continuously monitored at different wavelengths by instruments on the ground (e.g. Kanzelhöhe Flare Patrol service (Otruba, 1999)) and in space (e.g. GOES/XRS: Heckman et al., 1996; Balch, Hill, and Pizzo, 2002).

While flux increase as a consequence of a flare is relatively most significant in the $\mathrm{X}$ ray bandwidth, solar observations in EUV $(10-100 \mathrm{~nm} / 100-1000 \AA)$ also provide a good means to monitor solar-flaring activity in the transition region and lower corona.

SWAP onboard PROBA2 (Seaton et al., 2012; Halain et al., 2010, 2012; Berghmans et al., 2006) is a small EUV imager, imaging the solar corona in a bandpass centred around $17.4 \mathrm{~nm}(174 \AA)$. SWAP is a normal-incidence telescope in which the spectral selection is achieved by aluminium filters and multilayer coatings on the mirrors. Such a design provides a lighter and smaller instrument than can be achieved for grazing incidence telescopes, which can image the solar corona in X-rays. The $17.4 \mathrm{~nm}$ bandpass was chosen among alternative EUV options as it yields the strongest coronal signal, allowing for a smaller aperture and further reduction of the instrument size. SWAP was thus an exercise in miniaturisation; to build the smallest coronal imager still capable of imaging all space-weather relevant phenomena in the solar corona.

In this article we explore SWAP's utility as a solar-flare monitor. Can we build an operational flare-reporting service based on SWAP image data, while the SWAP $17.4 \mathrm{~nm}$ bandpass does not have a strong flare line such as the $19.5 \mathrm{~nm}(195 \AA)$ bandpass of EIT? The latter contains the strong Fe XXIV line, formed at $20 \mathrm{MK}$ during flares. This means that when plasma is heated during a solar flare, it can be heated so quickly that it is invisible in SWAP images.

Several EUV imaging telescopes with better resolution have been developed for scientific solar spacecraft, e.g. SOHO/EIT (Delaboudinière et al., 1995), TRACE (Golub et al., 1999), STEREO/SECCHI-EUVI (Aschwanden et al., 2009), and SDO/AIA (Lemen et al., 2012). With this study, however, we want to show that the resolution provided by state-of-the-art scientific instrumentation is not needed for space-weather monitoring and forecasting. In contrast, near real-time space-weather services have stronger requirements on the timeliness of data delivery and data processing, in order to provide warnings and alerts.

Flare identification in SWAP images is hindered by external effects such as the South Atlantic Anomaly (SAA). Flare-detection software is very sensitive to increased image noise generated by energetic particles impinging on the SWAP detector when the orbiter travels through the SAA region. We also learned that a small fraction of SWAP images are blurred by spacecraft motion, possibly influencing flare detection, and that SWAP's sometimes irregular cadence creates yet an extra challenge in the detection of rapidly evolving events. 
Why use SWAP images to detect flares? The prime focus of this article will be on a detection algorithm that automatically locates flares in the SWAP EUV time sequences in near real time. The output of our flare-detecting software is in the first instance intended as a service to the Space Weather Segment of ESA's Space Situational Awareness (SSA) program. It is a clear advantage being able to provide such a service making use of a small EUV imager.

We also consider the PROBA2/SWAP images as a model for the EUI data expected from the future Solar Orbiter mission. Because it is a deep-space mission, the latter mission will have severely restricted telemetry bandwidth. Therefore it is necessary to develop an onboard intelligence that can recognise the time and location of major solar eruptions. SWAP images provide a particularly good test platform for this algorithm since SWAP and EUI are both based on a CMOS/APS detector imaging in the $17.4 \mathrm{~nm}$ bandpass at an imaging cadence of the order of a minute (De Groof et al., 2008). More details regarding the onboard application will be subject of a future article.

Detection tools similar to the one that we will propose below have been developed for other instruments. The SDO Flare Detective (Grigis et al., 2010) uses EUV images that are in the first instance segmented into a small number of macropixels. Flare start times are determined by thresholding values of the first derivative of the lightcurve computed per macropixel. Flare end times are determined when the flux is lower than a certain fraction of the peak flux. The Hinode team applies their FLare Detector tool (FLD: Kano et al., 2008) to full-frame XRT images (Kosugi et al., 2007; Golub et al., 2007) taken at intervals of about 30 seconds and then in the first instance divided into $16 \times 16$ blocks (macropixels). An increase of X-ray intensity per macropixel is measured by taking the relative difference between an image and its reference image (the latter based on a weighted average of the previous images). A flare start is then defined by thresholding the differences. The RHESSI Flare finder (Lin et al., 2002) searches for microflares as local maxima in the $6-12 \mathrm{keV}$ count-rate timelines surrounded by episodes of positive and negative temporal derivatives. The algorithm analyses individual orbits separately. The event counts must be greater than three $\sigma$ of the noise of the global background counts and flares are limited to a maximum flare duration imposed by the RHESSI orbit.

A short description of the SWAP instrument and the characteristics of its EUV images is given in the next section. The detection algorithm is described in more detail in Section 3. Subsequently, statistical results from the application of the algorithm on the selected dataset are presented in Section 4. An online display in real time makes our tool's results accessible for the broad audience, as explained in Section 5. In Section 6 we formulate our conclusions, and we pose some ideas for further improvement and exploitation of the detection software.

\section{Input Dataset}

\subsection{SWAP EUV Imaging}

SWAP captures EUV images of the low solar corona in a bandpass peaking at $17.4 \mathrm{~nm}$ $(174 \AA$ ). When the instrument is operating in its nominal imaging mode, SWAP provides images over a $54 \times 54$ arcmin field-of-view with 3.17 arcsec pixels. The typical imaging cadence is around 130 seconds. The data are processed as soon as they arrive on the ground and are immediately distributed online as uncalibrated level-0 FITS files (Ponz, Thompson, and Munoz, 1994), via proba2.oma.be/data/SWAP. 


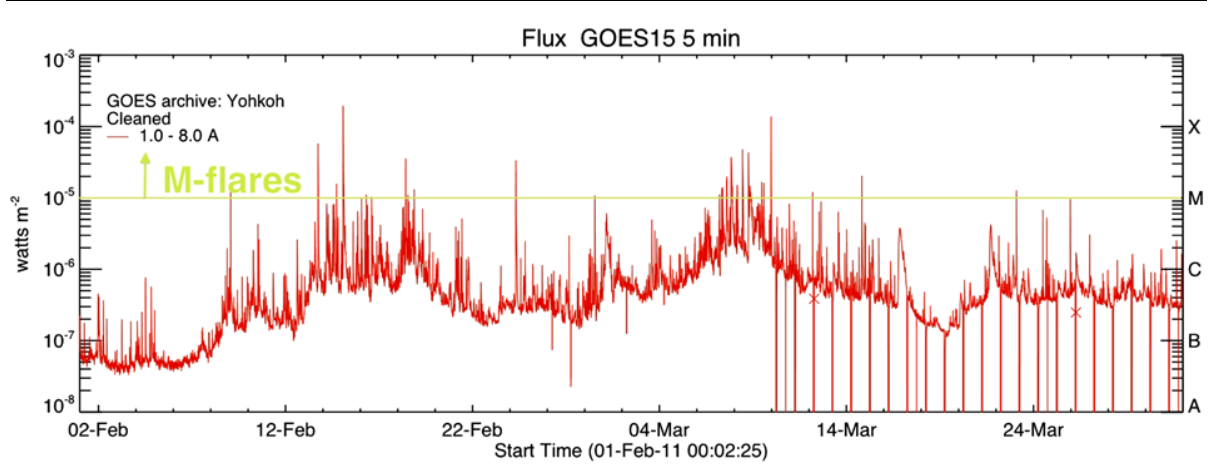

Figure 1 GOES X-ray flux (five-minute cadence data) corresponding to our dataset of February and March 2011. Our detection tool searches in the first instance for M-flares or stronger, i.e. flares peaking above the yellow horizontal line. Note: the vertical lines appearing in the GOES curve are the result of short data gaps in March 2011.

Once they are available, dedicated software in the PROBA2 Science Centre (Zender et al., 2012) automatically converts level-0 images into calibrated level-1 images. These images are dark subtracted and corrected for bad pixels as well as for telescopic and spacecraft effects so that the Sun is located in the centre of the image frame and solar North is at the top of the image. Level-1 images are normalised to the exposure time. The final data are given as Data Number per second per pixel [DN s ${ }^{-1}$ pixel $\left.^{-1}\right]$ in the FITS file format. Additional details regarding the calibration steps are given by Seaton et al. (2012).

\subsection{Data Selection}

In this article we used a test dataset consisting of about two months of SWAP data obtained in February and March 2011. These were among the first months of the current solar cycle in which there was significant solar activity. Figure 1, which shows GOES observations of solar flux in the 1.0-8.0 $\AA$ passband, illustrates the relatively active solar conditions during the period.

For our study on flare detection, we do not consider SWAP observations from special campaigns (e.g. off-pointing of the SWAP telescope). We work with a series of standard images, during which GOES identified 29 M-flares and one X-flare.

\section{Detection Software}

We describe here our flare-detection tool, which we refer to as the Solar Flare Automated Search Tool (SoFAST). We describe the algorithm developed for a space-weather monitoring service.

\subsection{The Flare Finding Algorithm}

Similar to other automated feature recognition tools, our algorithm consists of thresholdbased detection of brightness variations, clustered in space and time in image sequences. The algorithm, which is kept as simple as possible to limit the computation power needed, has seven main steps. The first six steps are illustrated in Figure 2. The last step concerns clustering events. 

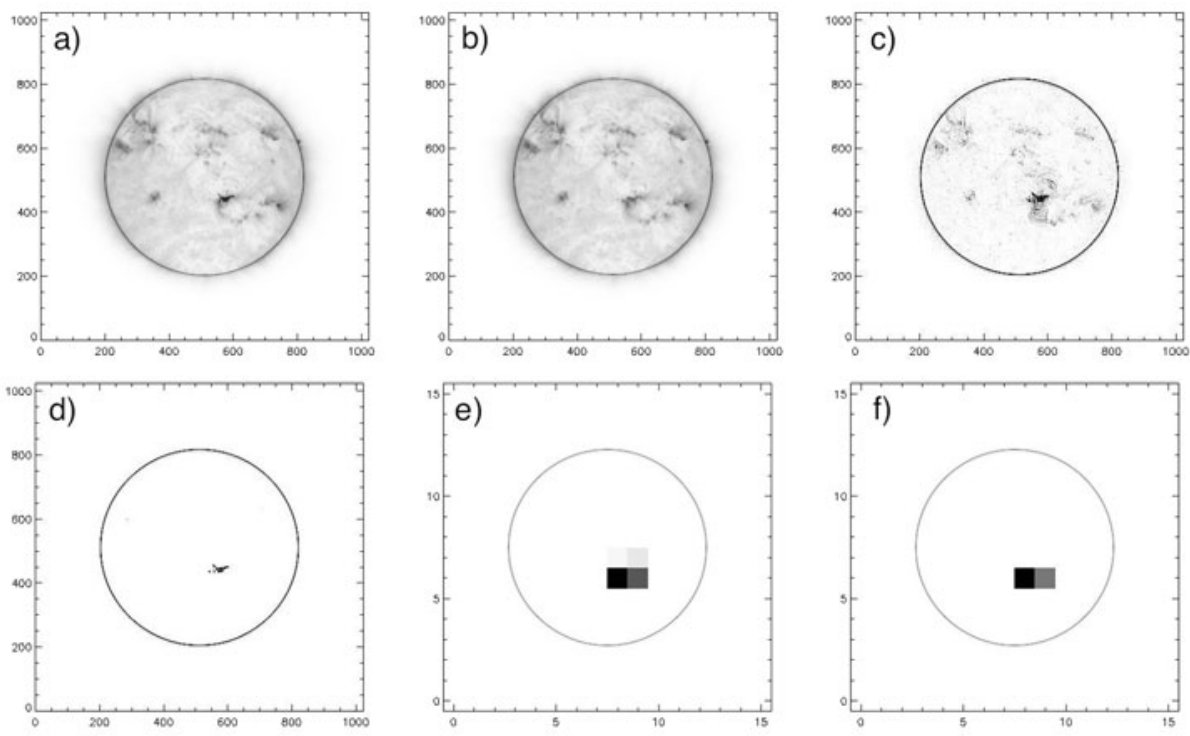

Figure 2 Images in reversed greyscale, illustrating the main steps of our algorithm for identifying a flare in a SWAP image (a). A second image (b) is identified 12 minutes prior to image (a). The absolute value of the running difference of (a) and (b) is taken, resulting in image (c). Images (d), (e), and (f) show subtracting the offset, rebinning, and thresholding the result, respectively. Solar limb position enhanced for reference.

i) Running-difference sequences.

Instead of trying to detect flares in full EUV images, we will search for intensity variations by making use of running-difference images. We compute these differences between SWAP images separated by about 12 minutes (with a tolerance of two minutes).

ii) Absolute value.

We then compute the absolute values of the resulting difference images. The result is a measure of absolute dynamic variation from image-to-image, regardless of whether it is an intensity increase (due to flare rise) or decrease (during flare decay, for example).

iii) Subtracting offset.

Only sufficiently significant variations in the difference images are retained by subtracting an offset value (and then continue working with the positive values); and thus a first level of thresholding is introduced. This enables us to eliminate values associated with the quiet Sun. The higher the offset parameter value, the more selective the detection.

iv) Rebinning.

Before thresholding, we segment the resulting difference images in a small number of "macropixels" by applying a rebinning operation. This operation implies neighbourhood averaging in order to degrade the data to a lower resolution. For the purposes of this article, we reduce the $1024 \times 1024$ images to $16 \times 16$ macropixels. With a pixel size of 3.17 arcseconds, one macropixel is 202.88 arcseconds on a side. Rebinning the images significantly reduces the computational power needed in the following steps. Since we are interested in the temporal behaviour of flaring regions, we stack our runningdifference $[x, y]$ images into an $[x, y, t]$ datacube. 
v) Thresholding to exclude false detections.

We apply two types of thresholding. The first type limits the number of false detections by excluding images with a large number of detections of extremely large variations in signal level. Images with many detections are frequently the result of a particle storm rather than of a flare. A so-called particle storm occurs, for example, when crossing the South Atlantic Anomaly (SAA), resulting in a high level of radiation noise in the images taken at that time. To restrict the necessary computational power, we consider this a sufficient method for excluding detections of cosmic rays or blurriness.

vi) Thresholding for flare selection.

During this step the algorithm identifies macropixels in which the signal exceeds a given threshold. We say the macropixel corresponds with a "hit" when the signal shows a "peak". The particular threshold selected for the algorithm corresponds to a minimum flare amplitude. In order to determine the 'borders' of a flaring event in space as well as in time, a slightly lower threshold is accepted for neighbouring values of a peak, once the peak is detected.

vii) Clustering events.

Points of interest in an $[x, y, t]$ datacube, i.e. points with peak values, are grouped together using clustering and morphological closing operations to mark out different events. The threshold for peak-neighbouring values obviously has an influence on two events being erroneously merged with one another, or conversely, numerous events being detected where one (big) event occurs. After grouping, we label different clusters as different dynamic events, assigning an event number to each detection.

Thresholds and other free parameters can be changed in order to optimise the desired output, depending on the application of the SoFAST tool. Table 1 lists the free parameters.

\subsection{Output}

When given a sequence of data, the program will run over all images and identify a set of events. For each event found, summary data are catalogued, including event characteristics as listed in Table 2. The first seven output-parameter names are self-explanatory. The eighth parameter, called EUV-significance, gives an idea of the importance of the event. It is defined as the intensity variation observed in the principal macropixel of the flaring region, in relation to the averaged macropixel intensity considering one complete day of observation (EUV relative variability). The principal macropixel [MP] of an event is the macropixel

Table 1 Free parameters/thresholds of SoFAST.

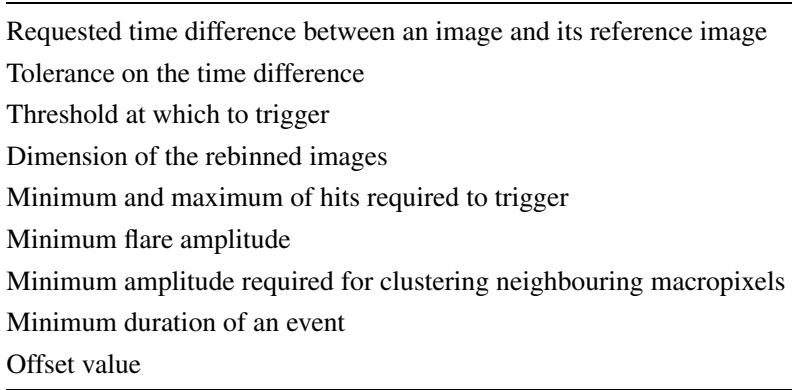


containing the highest intensity variation.

$$
\text { EUV-significance }=\frac{\max \left(I_{\mathrm{MP}_{\text {event }}}\right)-\min \left(I_{\mathrm{MP}_{\text {event }}}\right)}{\left\langle I_{\mathrm{MP}}\right\rangle},
$$

where $I_{\mathrm{MP}_{\text {event }}}$ is the EUV intensity observed during the concerned event in the corresponding principal macropixel, and $\left\langle I_{\mathrm{MP}}\right\rangle$ is the average EUV intensity observed in the same macropixel during the complete day.

The challenge is to identify flaring events without relying on comparison with the GOES $\mathrm{X}$-ray flarelist. This is very difficult given the intrinsic differences between X-ray observations and those in EUV. The EUV-significance parameter provides a first indication of the most dynamic EUV events. With some exceptions, most significant flaring events typically show an EUV-significance value of at least $8.0 \%$. An example of characteristics of one particular event is given in Table 2.

A typical visualisation of the output of SoFAST is illustrated in Figure 3. We give the example of the SoFAST detected event listed in Table 2. This flaring event corresponds with

Table 2 Output from our SoFAST detection tool: characteristics catalogued for one particular event that occurred during 7 March 2011.

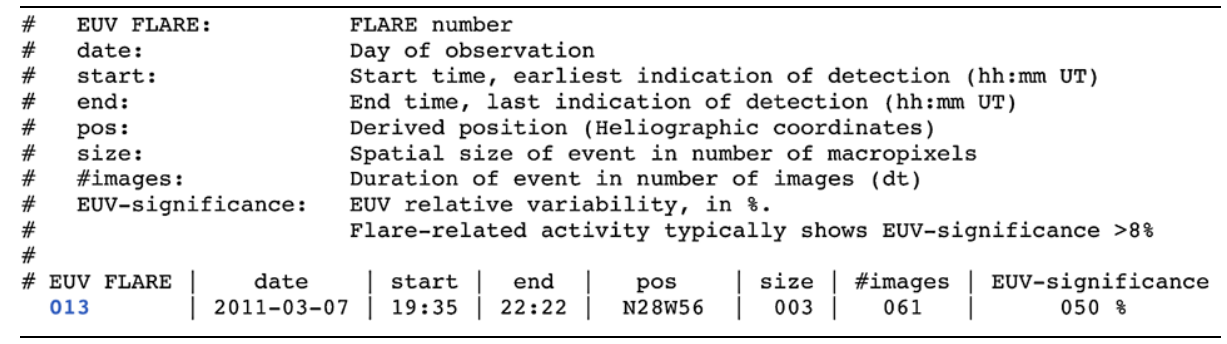
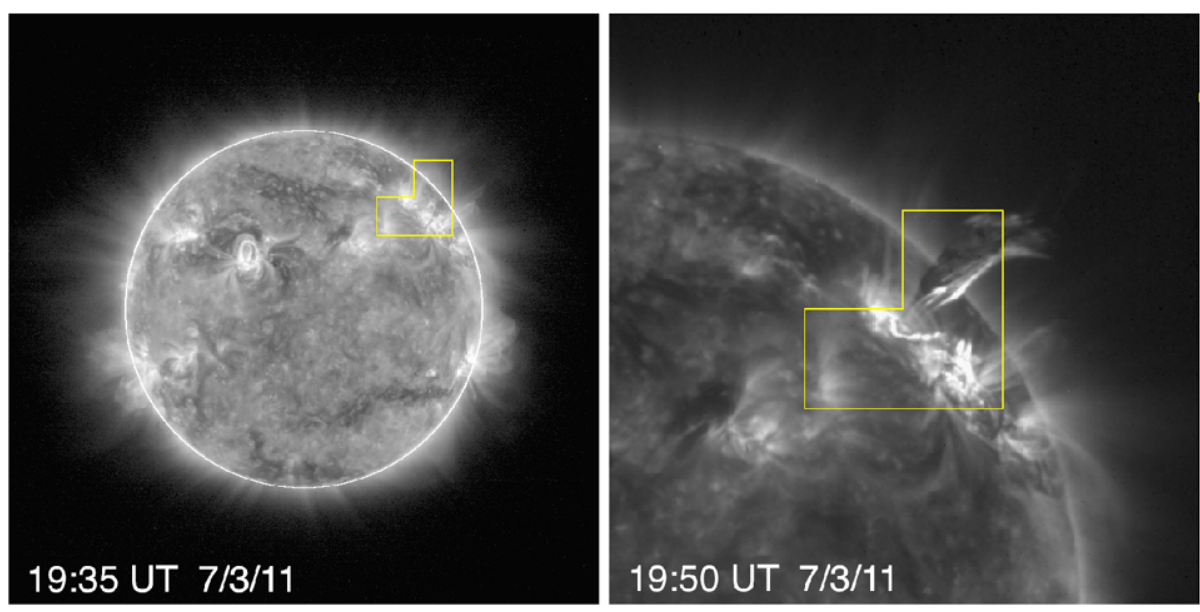

Figure 3 Visualisation of a particular SoFAST detection on 7 March 2011 (see characteristics listed in Table 2). This EUV-flaring event corresponds with the GOES M3.7 flare that occurred (peaked) on 7 March at 20:01 UT. Left: SWAP full solar disk image at the beginning of the flaring event (19:35 UT). The flaring region is marked in yellow, covering in this case three macropixels. One macropixel is 202.88 arcseconds on a side. Solar limb position enhanced for reference. Right: Close-up view of this SoFAST EUV flare detection, sample image at 19:50 UT. 


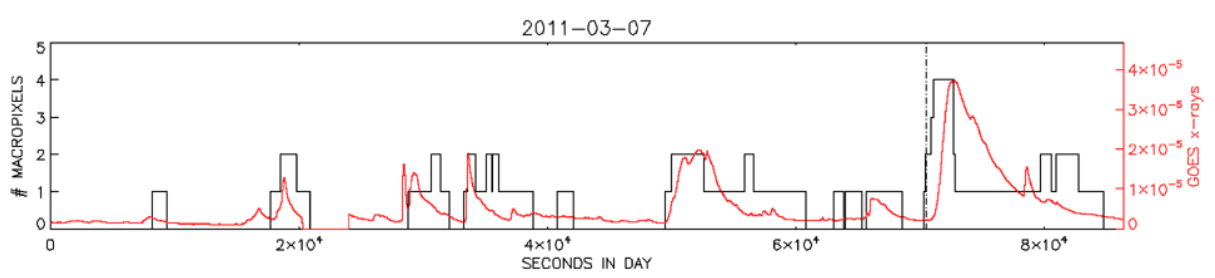

Figure 4 SoFAST EUV flare detections (in black, region size in macropixels) with GOES X-ray flux (red, arbitrary units) for observations on 7 March 2011. The horizontal axis gives observation time over the day, in seconds. The vertical axis represents the number of macropixels that cover the detected event. The dashed line highlights the time of the SoFAST detection that was illustrated in Figure 3.

the GOES M3.7 flare that occurred (peaked) on 7 March at 20:01 UT. The left panel shows the full solar disk at the start time of the flaring event (19:35 UT), with the macropixels covering the detected area marked with a coloured contour. The right panel shows a closeup view of the region of interest (sample image at 19:50 UT). When used for space-weather operations, the algorithm automatically generates a link to a running-difference movie of the close-up view of the detected region. Such a movie reflects how dynamic our detected flaring region really is in the EUV.

As the GOES X-ray monitor is currently still the most commonly used reference for flare reporting, we validate the output of SoFAST by comparing its EUV flare detections to the observed GOES X-ray flux. We illustrate this for the event of 7 March 2011 in Figure 4. Recall that we concentrate only on M-flares or stronger.

\section{Results: Statistics}

First of all, it is important to understand that the output of our SoFAST detection tool is influenced by the choice of parameters (thresholds) as listed in Table 1. For this study, we optimised the thresholds such that output would serve for space-weather forecast and alert services. This implies that the values of thresholds, based on a trial-and-error search of the parameter space, are chosen in order to maximise the number of GOES M-flares or stronger EUV flux variation that we detect.

\subsection{Comparison with GOES Flare Detections}

We will validate the output of SoFAST in Section 4.1.1 by verifying how well the distribution of detected EUV flares correspond to the distribution of GOES-detected M-flares or stronger. Note that, in general, X-ray flares do not necessarily correspond one-to-one to EUV flares. We also detect dynamic events that are not registered as a GOES flare, but nevertheless are of significant value. We will further refer to such events as "non-GOES events".

\subsubsection{A Typical One-Day SoFAST Detection}

As an example of SoFAST's output, we studied the characteristics of flaring events detected during one typical day: 8 March 2011. A summary of GOES detections of M-flares or stronger for that day is listed in Table 3. Together with the characterising parameters, we give an interpretation of the SoFAST detection in relation to the GOES flarelist in Table 4. 
Table 3 GOES M- and X-flares on 8 March 2011.

\begin{tabular}{ll}
\hline 02:24 UT-02:32 UT & M1.3 \\
$03: 37$ UT-04:20 UT & M1.5 \\
10:35 UT-10:55 UT & M5.3 \\
18:08 UT-18:41 UT & M4.4 \\
19:46 UT-21:19 UT & M1.4 \\
\hline
\end{tabular}

Table 4 SoFAST EUV flare detections for 8 March 2011. Parameters are listed as explained in Table 2, subsequently we give an interpretation of the observed events, comparing with the GOES flarelist. Events numbers 1, 2, 3, 6, 7, 11, 12, 14, and 16 show an EUV-significance value above $8.0 \%$. Event number 11 is the subject of further discussion in Section 4.1.2

\begin{tabular}{|c|c|c|c|c|c|c|c|c|c|c|}
\hline \multirow[t]{2}{*}{ \# } & \multirow[t]{2}{*}{ EUV FLARE } & \multirow{2}{*}{$\begin{array}{c}\text { date } \\
2011-03-08\end{array}$} & \multirow{2}{*}{$\begin{array}{l}\text { start } \\
23: 37\end{array}$} & \multirow{2}{*}{$\begin{array}{l}\text { end } \\
23: 55\end{array}$} & \multirow{2}{*}{$\begin{array}{c}\text { pos } \\
\text { N28W56 }\end{array}$} & \multirow{2}{*}{$\begin{array}{r}\text { size } \\
001\end{array}$} & \multirow{2}{*}{$\begin{array}{l}\text { \#images } \\
006\end{array}$} & \multicolumn{3}{|c|}{ EUV-sign. } \\
\hline & & & & & & & & 008 & 8 & nOn-GOES \\
\hline & 015 & $2011-03-08$ & $22: 32$ & $23: 55$ & S19W85 & 002 & 026 & 005 & \% & GOES C8.8 \\
\hline & 014 & $2011-03-08$ & $22: 07$ & $22: 42$ & N28W56 & 001 & 011 & 008 & 8 & nON-GOES \\
\hline & 012 & $2011-03-08$ & $20: 27$ & $21: 18$ & S19W85 & 001 & 027 & 020 & 8 & GOES M1.4 \\
\hline & 011 & $2011-03-08$ & $18: 57$ & $19: 22$ & N11W06 & 001 & 013 & 049 & 웅 & nOn-GoES \\
\hline & 010 & $2011-03-08$ & $18: 50$ & $19: 12$ & N28W56 & 001 & 011 & 005 & 8 & nOn-GOES \\
\hline & 007 & $2011-03-08$ & $10: 38$ & $11: 03$ & S19W85 & 001 & 014 & 008 & 응 & GOES M5.3 \\
\hline & 006 & $2011-03-08$ & $09: 32$ & $09: 37$ & S23W52 & 001 & 004 & 009 & 8 & GOES C4.7 \\
\hline & 005 & $2011-03-08$ & $06: 15$ & $06: 22$ & S23W52 & 001 & 004 & 002 & 8 & nON-GOES \\
\hline & 004 & $2011-03-08$ & $05: 32$ & $05: 52$ & S19E85 & 001 & 008 & 004 & $\%$ & nOn-GOES \\
\hline & 003 & 2011-03-08 & $03: 37$ & $05: 27$ & S19E85 & 001 & 039 & 031 & $\%$ & GOES M1.5 \\
\hline
\end{tabular}

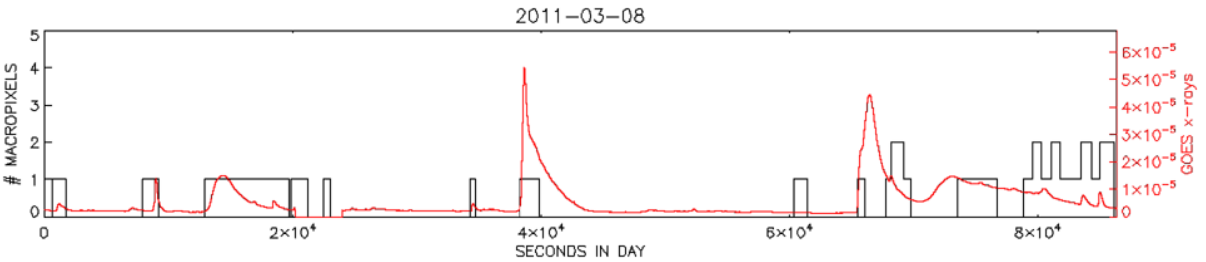

Figure 5 SoFAST EUV flare detections (in black, region size in pixels) with GOES X-ray flux (red, arbitrary units) for observations on 8 March 2011. The horizontal axis gives observation time over the day, in seconds. The vertical axis represents the number of macropixels that is covering the detected event.

Note that all of the GOES M- or X-flares are also SoFAST detections. In addition to these five detections, SoFAST detects two C-class flares and nine non-GOES events. The nonGOES events are not false alerts; often they are found to be associated with post-flare loops. Some cases are more dynamic; in Section 4.1.2 we describe one such case in detail. Figure 5 provides a comparison between SoFAST detections and GOES X-ray flux on 8 March 2011.

In Section 4.1.2 we give a first statistical interpretation of how well the SoFAST detections match the GOES flarelist, working with a dataset not of one day, but of two months showing significant solar activity.

\subsubsection{First Statistics from a Two-Month Dataset}

For a statistical assessment, we work with the full two-month dataset covering February and March 2011, as described in Section 2.2. A straightforward comparison of the output of our detection tool with GOES flare detections for this period is illustrated in the histogram of Figure 6. For the moment, we still focus only on the detection of M- and X-flares. 
Figure 6 Histogram illustrating the number of SoFAST EUV flare detections and the number of GOES flare detections for the period incorporating February and March 2011. We focus only on M- and X-flares.

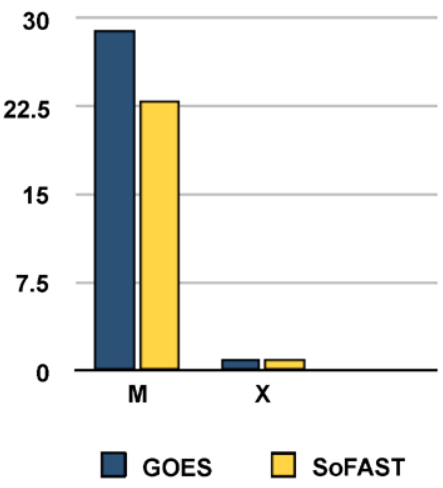

Table 5 Contingency table concerning SoFAST detections and GOES detections, for February and March 2011.

\begin{tabular}{lccc}
\hline & Observed in SWAP EUV & Not seen in SWAP EUV & Total \\
\hline $\begin{array}{l}\text { Observed in GOES X-rays } \\
\text { M+X class }\end{array}$ & $\mathbf{2 4}$ & $\mathbf{6}$ & $\mathbf{3 0}$ \\
$\begin{array}{l}\text { Observed in GOES X-rays } \\
\text { B+C class }\end{array}$ & 79 & 195 & 274 \\
$\begin{array}{l}\text { Not seen in GOES X-rays } \\
\text { EUV-significance } \geq 8 \%\end{array}$ & $\mathbf{5 2}$ & - & $\mathbf{5 2}$ \\
$\begin{array}{l}\text { Not seen in GOES X-rays } \\
\text { EUV-significance }<8 \%\end{array}$ & 65 & - & 65 \\
Total & 220 & 201 & 421 \\
\hline
\end{tabular}

SoFAST also detects EUV dynamical events that are not seen in X-rays. We illustrate with a contingency table (Table 5) how the complete list of SoFAST detections is distributed in comparison with GOES-detected M- or X-flares, still considering the same two-month period.

Recall that we do not take into account periods of SWAP special campaigns, where for example the SWAP telescope has been off-pointed. Also, we focus on M- and X-flares and non-GOES events with a high EUV-significance value.

We conclude that SoFAST detects on average $80 \%$ of GOES M- or X-flares (24 out of 30 ), which is good for an EUV flare detector facing many challenges as stated in the introduction. Additionally, interesting non-GOES dynamic events are detected as well. Thresholding on an EUV-significance of $8.0 \%$ refines the non-GOES events typically to the most interesting $45 \%$.

Here we give an example of one such non-GOES event. The event occurred in a region that we interpret as an EUV-flaring region on 8 March 2011, between 18:57 UT and 19:22 UT, located in the Northwest (central) on the solar disk. It shows an EUV-significance of $49 \%$. GOES does not register any flare before 23:10 UT in that area (NOAA Region 1166), nor has a GOES data gap been reported. Table 4 lists the characteristics of this nonGOES event EUV flare number 11), four illustrative output images are given in Figure 7.

We find that this non-GOES event is most likely associated with the occurrence of a CME. The detection by SoFAST of EUV dynamic activity appears to be linked to the eruption of a filament located slightly to the West of AR 1166, as shown in Figures 8(a) and (b). 

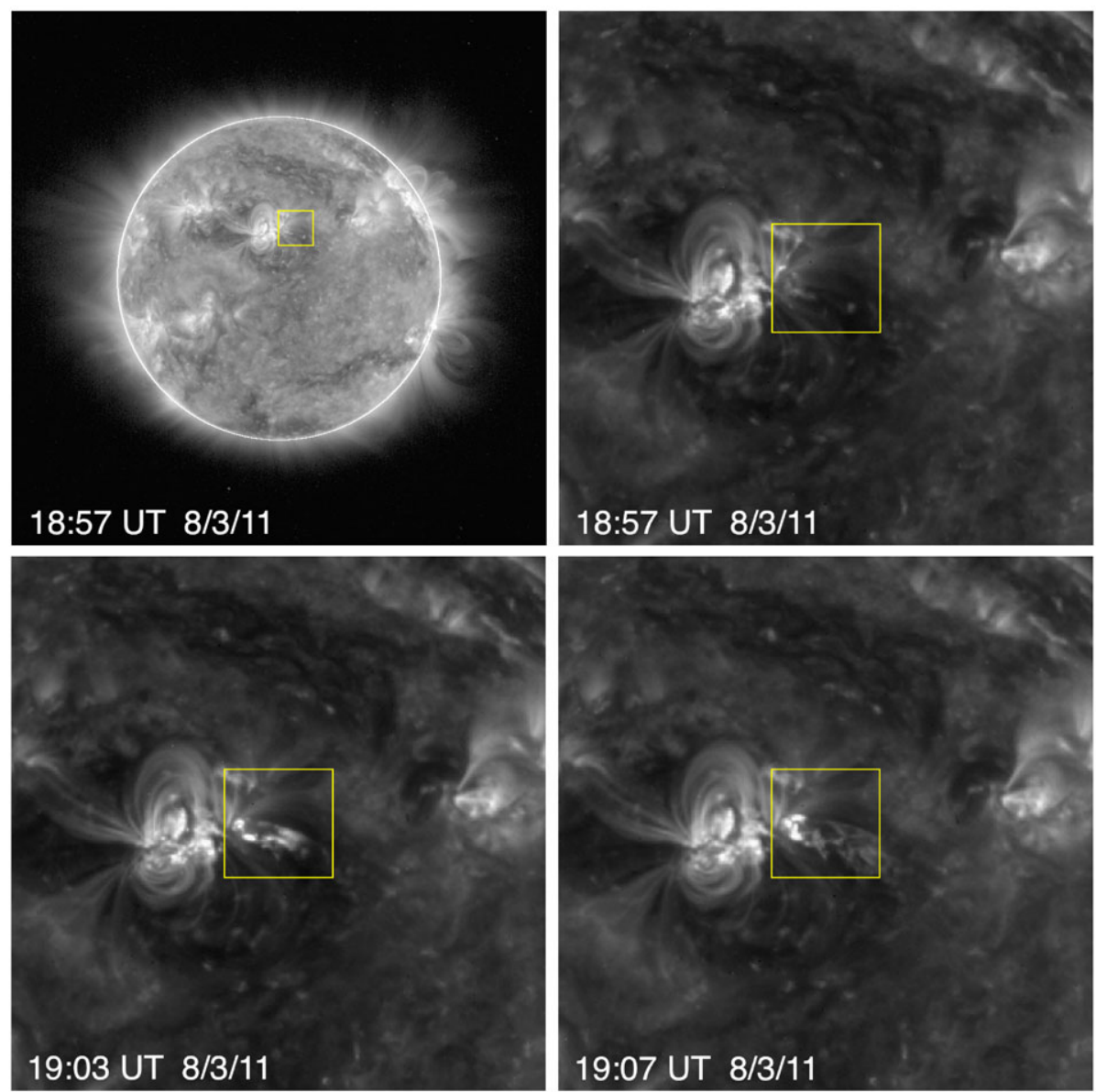

Figure 7 The upper-left panel shows the SoFAST non-GOES event detection at 18:57 UT on 8 March 2011 (event number 11 as previously listed in Table 4). The yellow box highlights the region of interest, covering in this case one macropixel. One macropixel is 202.88 arcseconds on a side. Solar limb position enhanced for reference. The other panels show the evolution of the flare occurrence in a close-up view at 18:57 UT, 19:03 UT, and 19:07 UT, respectively. GOES does not register any flare, associated with this region, before 23:10 UT, nor has a GOES data gap been reported.

This CME can be most clearly observed on the solar limb by STEREO-A, which was located approximately in quadrature with the Earth at this time. Figure 8(c) shows STEREOA/SECCHI-EUVI $304 \AA$ observations of the evolution of the eruption between 19:06 and 19:26 UT. Later, this CME is observed by the STEREO-A/COR-2 (Howard et al., 2008) coronagraph. The median velocity of this CME recorded in the CACTus CME catalogue (sidc.oma.be/cactus/) is $416 \mathrm{~km} \mathrm{~s}^{-1}$, which corresponds well with an estimated CME onset time of approximately 19:00 UT.

\section{Real-Time Version Online}

In addition to the above application of SoFAST to a test-case dataset, we are also developing a real-time version of the tool. This will be made available to complete the space- 
Figure 8 Evidence of a CME eruption associated with the detection by SoFAST of EUV dynamic activity between 18:57 and 19:22 UT on 8 March 2011. Panel (a) shows $\mathrm{H} \alpha$ observations from the Kanzelhöhe

Observatory of a filament located slightly West of AR 1166 at 10:44 UT. This filament is no longer visible in $\mathrm{H} \alpha$ observations from the Big Bear Solar Observatory (BBSO) at 20:54 UT, shown in panel (b).

The location of this filament is in good agreement with the location of the activity detected by SoFAST with SWAP, shown in Figure 7, and appears to be the likely location of the $\mathrm{CME}$ eruption. This CME is most clearly observed at the limb with STEREO-A. Panel (c) shows the evolution of this eruption in EUVI $304 \AA$ data. The CME is also later observed by the STEREO-A/COR-2 coronagraph. The angular extent of this CME, as determined by CACTus and highlighted by the yellow lines, is shown in panel (e). This event is unrelated to a second $\mathrm{CME}$ extending southwards that is also observed in the COR-2 FOV at this time. Yellow boxes in panels (a) to (d) highlight the regions of interest for this $\mathrm{CME}$ eruption.
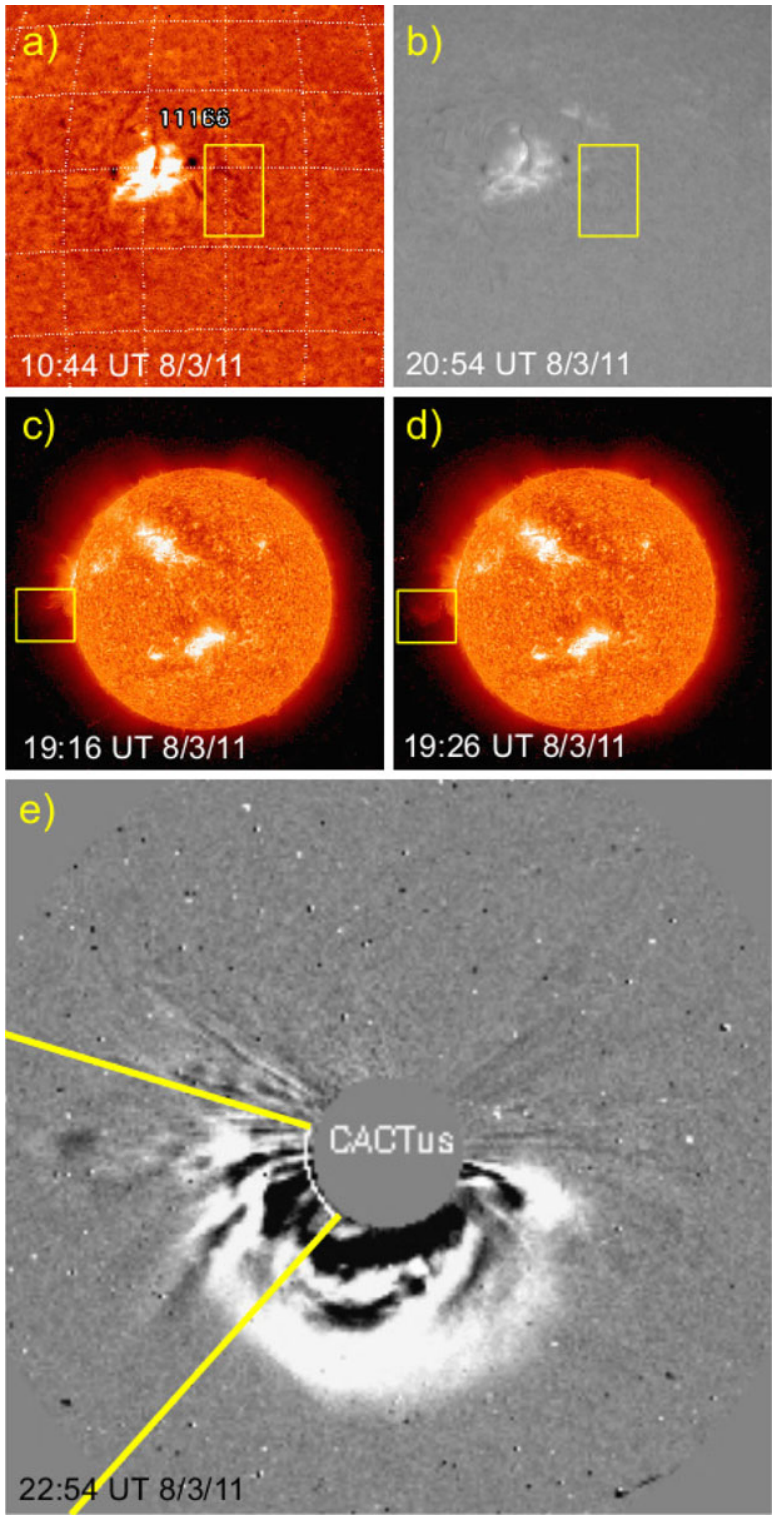

weather monitoring service through the Royal Observatory of Belgium (ROB) website (www.sidc.be). Triggered by a UNIX/crontab script, this real-time version automatically works with the latest SWAP level-1 images from the PROBA2 server. The output is an overview of EUV-flaring regions per day, indicated on a full-frame SWAP image, as well as a zoomed view of the actual dynamic region as shown above. Also a movie of the zoomed view is available when clicking on a flaring region. The performance and development of this real-time tool can be followed online at www.sidc.be/sofast. 


\section{Conclusions and Outlook}

In this article, we explained the different steps of our algorithm for detecting flares in EUV data from the PROBA2/SWAP imager. We demonstrated its capabilities in detecting Mand $\mathrm{X}$-flares in a test dataset and found our results compared favourably with the GOES $\mathrm{X}$-ray flarelist. In addition to the positive identification of high-energy flares, we detected a number of dynamic EUV events. We studied one so-called non-GOES event in detail and found that the detection is likely associated with a CME eruption observed in this region. Further investigation of these types of detection will be the subject of a future study.

Obtaining EUV data from SWAP that is suitable for flare monitoring can be difficult because of effects associated with the South Atlantic Anomaly (SAA), spacecraft motion, and SWAP's occasionally irregular cadence. However, despite the challenges of detecting flares in the EUV, we show that it is possible to deliver an operational flare-reporting service by means of our detection tool, SoFAST, making use of SWAP data. We conclude that SoFAST detects $80 \%$ of GOES M- or X-flares (24 out of 30) in a test dataset, which we consider a very good result for an EUV flare localiser.

A clear strength of SoFAST is its remarkable insensitivity to particle noise during the passage through the SAA and spacecraft jitter. This is possibly due to the use of macropixels and of the exclusion of detections showing an excessive number of "hits" in one image. Moreover, the defined EUV-significance parameter routinely quantifies the EUV relative variability, giving a first indication of EUV-flaring events autonomously. The robustness of the algorithm makes the SoFAST tool suitable for a variety of applications.

We have demonstrated the ability of the SoFAST algorithm to reliably detect M- and Xclass flares using only EUV observations. In the future, we intend to extend its capabilities to improve its suitability for multiple applications. Progressing towards an independent flaremonitoring service, our algorithm can best be complemented with results from a radiometer such as LYRA, ultimately resulting in a SWAP+LYRA joint space-weather monitoring tool. In addition, we plan to build a complete SWAP SoFAST catalogue. This will encompass both those events that are readily observed with GOES and those that we classified as nonGOES events. The EUV-significance parameter helps to define in an automated way how dynamic an active region really is (currently often verified manually). The latter parameter will be further investigated in order to categorise EUV dynamical events.

In the future, we anticipate that SWAP might also pave the way for the EUV Solar Imager for Operations: ESIO. This is a small, lightweight prototype solar EUV telescope (CMOS detector) that, together with a solar UV flux monitor, will be developed for the monitoring and forecasting of space-weather phenomena, comparable with SWAP and LYRA onboard of PROBA2. The results presented in this article on the EUV dynamics detecting capabilities of SoFAST are very relevant to the success of such a project.

With our software tool, we showed how interesting solar dynamic regions are detected in SWAP EUV images without requiring much computational power and with short notice. To this end, SWAP can be considered as a prototype for Solar Orbiter/EUI. The use of SoFAST for onboard detection is currently the subject of ongoing discussion. In terms of an onboard application, our algorithm can serve independently, as long as the number of "false alerts" remains low enough. The latter requirement can be fulfilled by applying stricter thresholds. Moreover, when aiming for a very selective detection, the robustness can be further increased by rebinning EUV images in an earlier stage in the detection process.

Acknowledgements These results were obtained in the framework of the projects GOA/2009-009 (KU Leuven), G.0729.11 (FWO-Vlaanderen) and C 90347 (ESA Prodex 9). The research leading to these results 
has also received funding from the European Commission's Seventh Framework Programme (FP7/20072013) under the grant agreements SOLSPANET (project no. 269299, www.solspanet.eu) and SWIFF (project no. 263340, www.swiff.eu) and eHEROES (project no. 284461). SWAP is a project of the Centre Spatial de Liège and the Royal Observatory of Belgium funded by the Belgian Federal Science Policy Office (BELSPO).

\section{References}

Aschwanden, M.J., Wuelser, J.P., Nitta, N.V., Lemen, J.R.: 2009, Solar flare and CME observations with STEREO/EUVI. Solar Phys. 256, 3-40. ADS:2009SoPh..256....3A, doi:10.1007/s11207-009-9347-4.

Balch, C.C., Hill, S.M., Pizzo, V.J.: 2002, Flare observations with the NOAA GOES-12 solar X-ray imager. In: AGU Fall Meeting Abstracts, A457.

Benz, A.O.: 2008, Flare observations. Living Rev. Solar Phys. 5(1). http://www.livingreviews.org/lrsp-2008-1.

Berghmans, D., Hochedez, J.F., Defise, J.M., Lecat, J.H., Nicula, B., Slemzin, V., et al.: 2006, SWAP onboard PROBA2, a new EUV imager for solar monitoring. Adv. Space Res. 38, 1807-1811. doi:10.1016/ j.asr.2005.03.070.

De Groof, A., Berghmans, D., Nicula, B., Halain, J.-P., Defise, J.-M., Thibert, T., Schühle, U.: 2008, CMOSAPS detectors for solar physics: lessons learned during the SWAP preflight calibration. Solar Phys. 249, 147-163. ADS:2008SoPh..249..147G, doi:10.1007/s11207-008-9175-y.

Delaboudinière, J.-P., Artzner, G.E., Brunaud, J., Gabriel, A.H., Hochedez, J.F., Millier, F., Song, X.Y., Au, B., Dere, K.P., Howard, R.A., Kreplin, R., Michels, D.J., Moses, J.D., Defise, J.M., Jamar, C., Rochus, P., Chauvineau, J.P., Marioge, J.P., Catura, R.C., Lemen, J.R., Shing, L., Stern, R.A., Gurman, J.B., Neupert, W.M., Maucherat, A., Clette, F., Cugnon, P., van Dessel, E.L.: 1995, EIT: Extreme-Ultraviolet Imaging Telescope for the SOHO mission. Solar Phys. 162, 291-312. ADS:1995SoPh..162..291D, doi:10.1007/BF00733432.

Golub, L., Bookbinder, J., Deluca, E., Karovska, M., Warren, H., Schrijver, C.J., Shine, R., Tarbell, T., Title, A., Wolfson, J., Handy, B., Kankelborg, C.: 1999, A new view of the solar corona from the Transition Region and Coronal Explorer (TRACE). Phys. Plasmas 6, 2205-2216. doi:10.1063/1.873473.

Golub, L., Deluca, E., Austin, G., Bookbinder, J., Caldwell, D., Cheimets, P., Cirtain, J., Cosmo, M., Reid, P., Sette, A., Weber, M., Sakao, T., Kano, R., Shibasaki, K., Hara, H., Tsuneta, S., Kumagai, K., Tamura, T., Shimojo, M., McCracken, J., Carpenter, J., Haight, H., Siler, R., Wright, E., Tucker, J., Rutledge, H., Barbera, M., Peres, G., Varisco, S.: 2007, The X-Ray Telescope (XRT) for the Hinode mission. Solar Phys. 243, 63-86. doi:10.1007/s11207-007-0182-1.

Grigis, P., Davey, A., Martens, P., Testa, P., Timmons, R., Su, Y., SDO Feature Finding Team: 2010, The SDO flare detective. Bull. Am. Astron. Soc. 41, 874.

Halain, J., Berghmans, D., Defise, J., Renotte, E., Thibert, T., Mazy, E., et al.: 2010, First light of SWAP on-board PROBA2. In: Soc. Photo-Optical Instr. Eng. CS 7732. doi:10.1117/12.857979.

Halain, J.-P., Berghmans, D., Seaton, D.B., Nicula, B., De Groof, A., Mierla, M., Mazzoli, A., Defise, J.-M., Rochus, P.: 2012, The SWAP EUV imaging telescope. Part II: In-flight performance and calibration. Solar Phys. doi:10.1007/s11207-012-0114-6.

Heckman, G., Speich, D., Hirman, J., Defoor, T.: 1996, NOAA Space Environment Center mission and the GOES space environment monitoring subsystem. In: Washwell, E.R. (ed.) Soc. Photo-Optical Instr. Eng. CS-2812, 274-280.

Howard, R., Moses, J., Vourlidas, A., Newmark, J., Socker, D., Plunkett, S., Korendyke, C., Cook, J., Hurley, A., Davila, J., Thompson, W., St Cyr, O., Mentzell, E., Mehalick, K., Lemen, J., Wuelser, J., Duncan, D., Tarbell, T., Wolfson, C., Moore, A., Harrison, R., Waltham, N., Lang, J., Davis, C., Eyles, C., MapsonMenard, H., Simnett, G., Halain, J., Defise, J., Mazy, E., Rochus, P., Mercier, R., Ravet, M., Delmotte, F., Auchere, F., Delaboudiniere, J., Bothmer, V., Deutsch, W., Wang, D., Rich, N., Cooper, S., Stephens, V., Maahs, G., Baugh, R., McMullin, D., Carter, T.: 2008, Sun Earth Connection Coronal and Heliospheric Investigation (SECCHI). Space Sci. Rev. 136, 67-115. doi:10.1007/s11214-008-9341-4.

Kano, R., Sakao, T., Hara, H., Tsuneta, S., Matsuzaki, K., Kumagai, K., Shimojo, M., Minesugi, K., Shibasaki, K., Deluca, E.E., Golub, L., Bookbinder, J., Caldwell, D., Cheimets, P., Cirtain, J., Dennis, E., Kent, T., Weber, M.: 2008, The Hinode X-Ray Telescope (XRT): camera design, performance and operations. Solar Phys. 249, 263-279. ADS:2008SoPh..249..263K. doi:10.1007/s11207-007-9058-7.

Kosugi, T., Matsuzaki, K., Sakao, T., Shimizu, T., Sone, Y., Tachikawa, S., Hashimoto, T., Minesugi, K., Ohnishi, A., Yamada, T., Tsuneta, S., Hara, H., Ichimoto, K., Suematsu, Y., Shimojo, M., Watanabe, T., Shimada, S., Davis, J.M., Hill, L.D., Owens, J.K., Title, A.M., Culhane, J.L., Harra, L.K., Doschek, G.A., Golub, L.: 2007, The Hinode (Solar-B) mission: an overview. Solar Phys. 243, 3-17. ADS:2007SoPh..243....3K, doi:10.1007/s11207-007-9014-6. 
Lemen, J.R., Title, A.M., Akin, D.J., Boerner, P.F., Chou, C., Drake, J.F., Duncan, D.W., Edwards, C.G., Friedlaender, F.M., Heyman, G.F., Hurlburt, N.E., Katz, N.L., Kushner, G.D., Levay, M., Lindgren, R.W., Mathur, D.P., McFeaters, E.L., Mitchell, S., Rehse, R.A., Schrijver, C.J., Springer, L.A., Stern, R.A., Tarbell, T.D., Wuelser, J.-P., Wolfson, C.J., Yanari, C., Bookbinder, J.A., Cheimets, P.N., Caldwell, D., Deluca, E.E., Gates, R., Golub, L., Park, S., Podgorski, W.A., Bush, R.I., Scherrer, P.H., Gummin, M.A., Smith, P., Auker, G., Jerram, P., Pool, P., Soufli, R., Windt, D.L., Beardsley, S., Clapp, M., Lang, J., Waltham, N.: 2012, The Atmospheric Imaging Assembly (AIA) on the Solar Dynamics Observatory (SDO). Solar Phys. 275, 17-40. ADS:2012SoPh..275...17L, doi:10.1007/s11207-011-9776-8.

Lin, R.P., Dennis, B.R., Hurford, G.J., Smith, D.M., Zehnder, A., Harvey, P.R., Curtis, D.W., Pankow, D., Turin, P., Bester, M., Csillaghy, A., Lewis, M., Madden, N., van Beek, H.F., Appleby, M., Raudorf, T., McTiernan, J., Ramaty, R., Schmahl, E., Schwartz, R., Krucker, S., Abiad, R., Quinn, T., Berg, P., Hashii, M., Sterling, R., Jackson, R., Pratt, R., Campbell, R.D., Malone, D., Landis, D., Barrington-Leigh, C.P., Slassi-Sennou, S., Cork, C., Clark, D., Amato, D., Orwig, L., Boyle, R., Banks, I.S., Shirey, K., Tolbert, A.K., Zarro, D., Snow, F., Thomsen, K., Henneck, R., McHedlishvili, A., Ming, P., Fivian, M., Jordan, J., Wanner, R., Crubb, J., Preble, J., Matranga, M., Benz, A.O., Hudson, H.S., Canfield, R.C., Holman, G.D., Crannell, C., Kosugi, T., Emslie, A.G., Vilmer, N., Brown, J.C., Johns-Krull, C.M., Aschwanden, M.J., Metcalf, T.R., Conway, A.: 2002, The Reuven Ramaty High-Energy Solar Spectroscopic Imager (RHESSI). Solar Phys. 210, 3-32. ADS:2002SoPh..210....3L, doi:10.1023/A:1022428818870.

Otruba, W.: 1999, High cadence digital full disk H $\alpha$ patrol device at Kanzelhöhe. In: Schmieder, B., Hofmann, A., Staude, J. (eds.) Third Advances in Solar Physics Euroconference: Magnetic Fields and Oscillations CS-184, Astron. Soc. Pac., San Francisco, 314-318.

Ponz, J.D., Thompson, R.W., Munoz, J.R.: 1994, The FITS image extension. Astron. Astrophys. Suppl. 105, $53-55$.

Seaton, D.B., Berghmans, D., Nicula, B., Halain, J.-P., De Groof, A., Thibert, T., Bloomfield, D.S., Raftery, C.L., Gallagher, P.T., Auchère, F., Defise, J.-M., D’Huys, E., Lecat, J.-H., Mazy, E., Rochus, P., Rossi, L., Schühle, U., Slemzin, V., Yalim, M.S., Zender, J.: 2012, The SWAP EUV imaging telescope part I: instrument overview and pre-flight testing. Solar Phys. doi:10.1007/s11207-012-0114-6.

Zender, J., Berghmans, D., Bloomfield, D.S., Cabanas Parada, C., Dammasch, I., De Groof, A., D’Huys, E., Dominique, M., Gallagher, P., Giordanengo, B., Higgins, P.A., Hochedez, J.-F., Yalim, M.S., Nicula, B., Pylyser, E., Sanchez-Duarte, L., Schwehm, G., Seaton, D.B., Stanger, A., Stegen, K., Willems, S.: 2012, The Projects for Onboard Autonomy (PROBA2) science centre: Sun Watcher using APS Detectors and Image Processing (SWAP) and Large-Yield Radiometer (LYRA) science operations and data products. Solar Phys. doi:10.1007/s11207-012-0033-6. 